Literatura y Lingüística $\mathrm{N}^{\circ} 25$

ISSN 0716-5811 / pp. 145-168

\title{
El desarrollo de la sintaxis en la adolescencia: posibles influencias de naturaleza social ${ }^{1}$
}

\author{
Carlos Balboa Espinoza* \\ Nina María Crespo Allende** \\ Marcela Rivadeneira Valenzuela**
}

\section{Resumen}

La variación lingüística a nivel sintáctico que se correlaciona con la clase social del hablante es interpretada de dos maneras distintas. Una (Whiteman y Deutsch 1968) propone que los hablantes de estratos bajos hablan peor que los de estratos altos, basándose en la teoría del déficit de Bernstein (1971). La otra corresponde a la visión variacionista (Labov 1965, 1966, 1972; Weinreich et al. 1968; Labov y Sankoff 1985) que considera ambas opciones como formas de decir lo mismo. A la luz de esta polémica, antigua pero todavía no resuelta, se compara la complejidad sintáctica de discursos narrativos de 60 jóvenes de estratos altos y bajos con el mismo nivel de instrucción. El discurso fue segmentado y medido utilizando la propuesta de las unidades -t (Hunt 1970; Véliz 1988). Los resultados indican que, a pesar de que los jóvenes de estratos altos producen textos más extensos, no hay diferencias significativas en la complejidad sintáctica. Se respalda, de esta forma, la propuesta variacionista, aunque todavía es necesario dar cuenta de la influencia del nivel de instrucción del hablante y el contexto estilístico del uso.

Palabras clave: complejidad sintáctica, discurso narrativo, teoría del déficit, variación sociolingüística.

\section{Syntactic development in adolescents: possible social influence}

\footnotetext{
Abstract

There are two different ways of interpreting the socially correlated linguistic variation on the syntactic level. The first (Whiteman y Deutsch 1968), based on Bernstein's deficit hypothesis (Bernstein 1971), proposes that low class speakers

1 Artículo enmarcado dentro del proyecto FONDECYT 1100600.

Magíster en Lingüística aplicada, Pontificia Universidad Católica de Valparaíso, profesorbalboa@ gmail.com

** Doctora en Lingüística, Pontificia Universidad Católica de Valparaíso, ncrespo@ucv.cl

**** Chilena, Doctora en Ciencias del Lenguaje y Lingüística Aplicada, Universidad Católica de Temuco,mrivadeneira@uct.cl
} 
El desarrollo de la sintaxis en la adolescencia: posibles influencias de naturaleza social / Carlos Balboa, Nina Crespo y Marcela Jonely de Lourdes Rivadeneira

show a poor level of language when compared with the high class speakers. The second view stems from the variationist paradigm (Labov 1965, 1966, 1972; Weinreich et al. 1968; Labov y Sankoff 1985) and considers this distinction as two different ways of expressing the same content. In this study, we compare the syntactic complexity in narratives of $60 \mathrm{high} / \mathrm{low}$ class adolescents with the same level of instruction. The narratives were segmented and analyzed using the T-unit proposal (Hunt 1970; Véliz 1988). The results indicate that-even though the high class speakers produce longer texts-there is no significant difference between the syntactic complexity of the two groups. Thus, the variationist view is supported, though it is still necessary to consider the effect of the level of instruction and the situational context of use.

Key words: syntactic complexity, narratives, deficit hypothesis, sociolinguistic variation.

Recibido: 30-11-2011 Aceptado: 25-01-2012

\section{Introducción}

El presente trabajo se inscribe en dos ámbitos. Por un lado, en la sociolingüística variacionista (Labov 1965, 1966, 1972, 1994, 2001; Labov y Sankoff 1985; Cedergren y Sankoff 1974; Sankoff 1978, 1988a) ya que busca indagar las diferencias lingüísticas que caracterizan a los diferentes grupos sociales. Estas variaciones han sido señaladas en los diferentes niveles de la lengua en la variedad del español de Chile por autores como Silva-Corvalán (1980-1981, 1989, 2001), Oyanedel y Samaniego (1998-1999) y Sáez (2000) y son la expresión de nuestra identidad como comunidad de habla. Por otro lado, nuestro trabajo se inscribe en el desarrollo tardío de la sintaxis oral (Hunt 1970; Berman 2000), entendido como un desenvolvimiento durante la edad escolar de la habilidad para establecer relaciones entre las cláusulas y las oraciones que constituyen un texto.

Ahora bien, desde la perspectiva sociolingüística, se sostiene que existen diversas formas de decir lo mismo y que la elección de una por sobre otra depende de factores tanto internos como externos (Moreno Fernández 1998; Silva-Corvalán 2001). Desde la dimensión interna, estas formas pueden adscribirse a diferentes niveles de la lengua: fonéticofonológico, sintáctico o léxico, mientras que, desde una dimensión externa, la variación se ve condicionada por ciertas variables, entre ellas, la edad, el sexo o la clase social a la que pertenece un individuo (Labov 1983). Concretamente, este estudio busca dar cuenta si existen variaciones sintácticas que puedan ser dimensionadas en cuanto a mayor

$+146$


o menor complejidad sintáctica en producciones orales de adolescentes de distintas clases sociales.

Se ha constatado que existen diferencias a nivel gramatical entre las clases sociales de Chile (Silva-Corvalán 1980-1981; Makuc 2008). Sin embargo el propósito de nuestra investigación tiene otro sentido: analizar dicha diferencia en términos de la complejidad sintáctica, lo cual nos permite vincular este estudio a una problemática relacionada con la manera en que deben entenderse las variaciones lingüísticas entre individuos de diversas clases sociales. En este sentido, algunos autores (Almeida 2003; Sankoff y Labov 1985; López Morales 1993; Moreno Fernández 1998; Trudgill 2000) consideran que no puede identificarse esta diferenciación en términos de mejor o peor manejo de la lengua, vale decir, los rasgos peculiares que tienen los estratos más bajos no pueden considerarse peores o más pobres que los de los estratos medios y altos, sino variantes de una misma forma. No obstante, en la creencia popular, el registro de los hablantes de estratos sociales bajos se considera erróneo o empobrecido, e incluso, a partir de la teoría de Bernstein $(1974,1989)$, se propone la idea de "privación verbal" o déficit, la cual sostiene que el fracaso escolar de los sujetos de estratos más bajos obedece a una suerte de déficit lingüístico, en comparación con aquellos sujetos pertenecientes a los estratos medios y altos. De las diversas variaciones provocadas por la clase social, interesa aquí estudiar el desarrollo de la complejidad sintáctica que se produce durante la edad escolar.

La relevancia de este tema radica en que permitirá comprender, de manera más acabada, de qué forma incide la variable socioeconómica en la complejidad sintáctica de narraciones orales producidas por sujetos de distintas clases sociales, cuestión que se ha resuelto sólo de manera apriorística y sin contar con datos empíricos que validen dichos planteamientos. A partir de allí, el presente artículo busca indagar si existe una diferencia entre la complejidad sintáctica en narraciones orales producidas por estudiantes de estrato socioeconómico alto y bajo de Valparaíso, Chile. Para lograr nuestro propósito, primero se desarrollarán los conceptos de variación sociolingüística y las propuestas para medir la sintaxis en sujetos en edad escolar en el ámbito anglo e hispanohablante, dando cuenta de los trabajos en el área. Luego, se detallará la metodología, se expondrán los resultados y la discusión final. 
El desarrollo de la sintaxis en la adolescencia: posibles influencias de naturaleza social / Carlos Balboa, Nina Crespo y Marcela Jonely de Lourdes Rivadeneira

\section{Marco Teórico}

\subsection{La variación sociolingüística}

Lewandowski (1995) propone que las variantes lingüísticas corresponden al uso de la lengua en situaciones específicas, en relación a las clases y/o grupos que interactúan, a las variedades interpersonales e intrapersonales, y a las funciones o propósitos que se quieren lograr. Además, existe un supuesto en el sentido de que la variación afecta al sistema de la lengua en su totalidad, es decir, en todos sus niveles: desde lo fonético-fonológico, hasta lo discursivo, pasando por lo gramatical y lo léxico (López Morales 1993). Sin embargo, este supuesto no está aceptado unánimemente. En este sentido, es necesario señalar que existe un fuerte debate en cuanto a considerar la variación solo a nivel fonético-fonológico, sin una clara identificación en los niveles léxicos y sintácticos (Lavandera 1984, Trujillo 1996). En este sentido, Silva-Corvalán (1980-1981, 2001) señala, al considerar las investigaciones de oraciones con clíticos pleonásticos en Santiago de Chile, que las formas que duplican el clítico y las que no lo duplican serían formas distintas de decir lo mismo, pero al nivel del análisis de factores pragmáticos no serían auténticas variantes, si los hablantes las utilizan con propósitos comunicativos diferentes. En resumen, podemos hablar de variación más allá del nivel fonológico, si existe la certeza de que no hay un propósito comunicativo que determine la alternancia de una variante $u$ otra. Además, dicha variación debe presentarse a nivel de lengua y estar determinada por variables sociales como edad, sexo o estrato social.

\subsection{Clase social y teoría del déficit de Bernstein en la variación lingüística}

El trabajo pionero de William Labov y su investigación en el habla de ciertas comunidades en Nueva York (1972) permitió establecer por primera vez una escala de clasificación social en la cual se consideraban factores como el nivel de instrucción, la ocupación y los ingresos familiares. Así, Labov logró determinar que existía un paralelismo entre la posición en la jerarquía social y la frecuencia de realización de ciertas variables lingüísticas. Asimismo, demostró que si una variante es prestigiosa, todos los estratos socioeconómicos la evalúan positivamente y viceversa, por lo que corresponde a un principio de uniformidad, lo que sirve de base para considerar a estos sujetos como pertenecientes a una comunidad de habla. 
A partir de lo anteriormente señalado, el constructo de clase social es el más utilizado en las investigaciones sociolingüísticas, por lo cual en nuestra investigación lo utilizamos para determinar la variable social, en tanto podemos establecer comparaciones con otras investigaciones que se han desarrollado en el mismo ámbito.

Íntimamente ligada a la variable clase social, se encuentran las investigaciones del sociólogo británico Basil Bernstein, quien, preocupado fundamentalmente por el proceso de socialización de los individuos, ha prestado atención al papel que juega el lenguaje, como sistema simbólico y social, en dicho proceso. Desde principios básicamente sicolingüísticos, este investigador ha puesto en relación el lenguaje con la clase social, la escolaridad y el contexto en el cual se desenvuelven los hablantes, lo cual le ha permitido desarrollar su "teoría del déficit" (Moreno Fernández 1998).

Desde una perspectiva whorfiana, la cual establece relaciones entre clase social, estructura de la lengua y estructura de la percepción, esto es, que la estructura social genera distintas formas lingüísticas o códigos que transmiten esencialmente la cultura y de esa manera delimitan el comportamiento, la teoría del déficit identifica dos formas de expresión lingüística, de uso de la lengua o códigos, las cuales se denominan código restringido y código elaborado. Bernstein (1985) aclara que su noción de código corresponde más bien a una noción de códigos sociolingüísticos en vez de códigos lingüísticos, por lo que el concepto de código sociolingüístico se referirá a la estructuración social de los significados y a sus manifestaciones lingüísticas en contextos diferentes, pero relacionados.

Tradicionalmente, se ha considerado a cada uno de los conceptos de códigos planteados por Bernstein como propio de una clase o estrato socioeconómico. Es así como el código restringido se plantea como predominante en las clases o estratos socioeconómicos bajos, mientras que el código elaborado se identifica como más propio de las clases medias y altas. Lo que busca Bernstein es más bien asociar los códigos por él planteados a estilos de interacción social, a procesos de cognición y a formas distintas de interpretar la estructura social. Para Bernstein, todos los hablantes, no importando su clase social, tienen acceso a un código restringido, el cual surge de los vínculos sociales más cercanos e inmediatos, pero solamente algunos grupos tienen acceso al código elaborado. De esta manera, una diferencia clara entre grupos o clases sociales será que algunos ocuparán, en las más diversas situaciones, un código restringido, mientras que otros lo reservarán solamente para situaciones familiares y privadas (Bernstein 1974, 1985). 
El desarrollo de la sintaxis en la adolescencia: posibles influencias de naturaleza social / Carlos Balboa, Nina Crespo y Marcela Jonely de Lourdes Rivadeneira

Bernstein (1985) identifica el código restringido como aquel que orienta y sensibiliza a los usuarios en significados particularistas, es decir, está más estrechamente ligado a una estructura social particular y se presta menos a un cambio de principios. En este sentido, el sujeto socializado toma menos conciencia de los fundamentos de su socialización y sus posibilidades de reflexión son más limitadas. Se puede agregar, además, que el código restringido tiene su fundamento en símbolos condensados, por lo que se basa fundamentalmente en la metáfora.

Por el contrario, el código elaborado orienta a sus usuarios hacia significados universalistas, por lo que está menos ligado a una estructura particular y conlleva la posibilidad de un cambio en los principios; en este caso, el habla puede ser liberada de la estructura social y adquirir cierta autonomía. Con un código elaborado, el sujeto socializado accede más fácilmente a la comprensión de los principios que inspiran su socialización y puede, de esta manera, efectuar una reflexión sobre el orden social que le ha sido transmitido. Por último, a diferencia del código restringido, el código elaborado tiene sus bases en símbolos articulados y se basa fundamentalmente en la racionalidad (Bernstein 1985).

La teoría de Bernstein ha ido evolucionando desde una manera muy estricta y whorfiana en sus primeros textos (dos códigos ligados a dos clases sociales y a dos tipos de percepción), hacia una perspectiva más ligada al funcionalismo de Halliday, en el cual los dos códigos son principios reguladores que generan distintas variantes del habla en distintos contextos. Pese a esto, son sus primeros trabajos los que han gozado de una mayor proyección y aceptación, tanto en el ámbito pedagógico como en el de la sociolingüística. Muchos consideran que su valor está en que sus trabajos, como los de quienes han continuado sus postulados teóricos, demuestran que socialmente existen diferencias lingüísticas (Fernández Lozano 1994).

En síntesis, la teoría del déficit postula que aquel niño que solo tiene acceso al código restringido, tenderá a desarrollarse sólo de acuerdo a las reglas propias de este código; y, si bien no se trata de que un código sea mejor que el otro, puesto que cada uno tiene sus propias posibilidades y su propia estética, señala explícitamente que el sujeto que utiliza el código restringido manifestará un habla que tendrá una organización sintáctica limitada (Bernstein 1974).

$+150$




\subsection{Propuestas para medir la sintaxis en el ámbito del inglés}

Según Manjón-Cabeza (2008), la evaluación de la madurez sintáctica en el ámbito escolar, en las escasas veces que es medida, se hace de manera impresionista, con un desconocimiento de análisis cuantitativos confiables. Los esfuerzos por subsanar esta situación, cuyo máximo exponente en lengua española es López Morales (1984), se han centrado en la aplicación y difusión del método ideado por Hunt (1970), a través de los índices de madurez sintáctica. En nuestra lengua, los trabajos que se han desarrollado tienen el mérito de haber adaptado esta técnica surgida para el análisis en lengua inglesa.

La búsqueda de unos índices objetivos y fiables del desarrollo sintáctico fue tarea prioritaria para autores como Kellog Hunt (1970) quien, a partir de la gramática generativa transformacional, propone los índices primarios y secundarios para medir la complejidad sintáctica. Para lograr su propósito establece dos unidades de análisis, la unidad mínima terminal o unidad -t y la cláusula. A la unidad mínima terminal o unidad -t, la denomina terminal en el sentido de que "es gramaticalmente aceptable iniciarla con mayúscula y terminarla con punto o signo de interrogación" (Hunt 1970: 4) y mínima porque es "la unidad más corta en que puede dividirse un texto sin dejar ningún fragmento de oración como residuo" (Hunt 1970: 4). Cada una de estas unidades consiste de "una cláusula principal más cualquier cláusula o cláusulas subordinadas que estén agregadas o incrustadas en ella" (Hunt 1970: 5). Por otra parte, la cláusula es descrita como "un sujeto o un conjunto de sujetos coordinados con un verbo finito o un conjunto finito de verbos coordinados" (Hunt 1970: 3).

A partir de allí, propone como índices la longitud en palabras de unidad -t, la de la cláusula y la cantidad de cláusulas por unidad -t o índice de subordinación (Hunt 1970). Los índices secundarios que propone Hunt (1970) se dividen en clausales y no clausales. Los clausales son el número de cláusulas sustantivas por unidad terminal, el número de cláusulas adjetivas por unidad terminal y el número de cláusulas adverbiales por unidad terminal. Los no clausales son bastante numerosos y consisten en una proporción de elementos sintagmáticos o de sintagmas por unidad terminal, tales como sintagmas preposicionales por unidad terminal, infinitivos por unidad terminal, adjetivos calificativos por unidad terminal, etc. Hunt (1970) plantea que estos índices se basan en los principios de la gramática generativa; su propuesta es que, desde las 
El desarrollo de la sintaxis en la adolescencia: posibles influencias de naturaleza social / Carlos Balboa, Nina Crespo y Marcela Jonely de Lourdes Rivadeneira

oraciones básicas, se llega a los otros tipos aplicando transformaciones simples. De esta manera, la extensión de las unidades -t no implica sólo la cantidad de palabras, sino que a medida que es más extensa una unidad -t, mayor es el número de transformaciones (en el sentido chomskiano del término) que posee.

Ahora bien, si bien la base teórica es la gramática generativa transformacional, el inspirador de esta idea no fue Chomsky, quien nunca pretendió hablar del uso del lenguaje, sino de su competencia. Las propuestas de Hunt (1970) se fundamentan en un artículo de Miller de 1962 (Junco 1999; Checa García y Lozano, 2002; Barrera y Fraca de Barrera 2004), en el cual se plantea la teoría de la complejidad derivacional o TDC. Miller (1962) considera que la gramática generativo-transformacional de 1957 representa un buen modo de describir la propiedad combinatoria de la lengua (elementos finitos y reglas finitas de combinación que dan lugar a combinaciones ilimitadas), por lo que tratará entonces de probar que esas combinaciones se dan en la mente de los hablantes en exactamente la forma descrita por la teoría. Así, las oraciones "básicas" o kernel son las representaciones mentales que el hablante tiene de sus oraciones y la producción oral o escrita tienen como punto de origen estas oraciones y la comprensión oral o lectora las tienen como punto de llegada. A partir de allí se postula la "hipótesis de codificación": la mayor o menor complejidad sintáctica dependerá del mayor o menor número de transformaciones (opcionales) a codificar o decodificar, a partir de estas oraciones básicas.

En resumen, a partir de un análisis de textos escritos producidos por estudiantes de diversos niveles y adultos, Hunt (1970) plantea que existe una relación entre edad, inteligencia y madurez sintáctica y que los estudiantes van complejizando su sintaxis escrita, lo que se observa en el incremento de las unidades -t, de las cláusulas y del índice de subordinación.

La propuesta de Hunt (1970) está en este momento siendo reemplazada por enfoques más textuales y funcionalistas de la sintaxis, como la propuesta de los paquetes clausulares (Katzenberger 2004; Nir y Berman 2010) que plantea una medición de la sintaxis más estrechamente ligada con el discurso. Sin embargo, hasta la fecha, ninguna de estas propuestas ha logrado la rigurosidad empírica del método de medición de Hunt (Majón Cabezas 2008).

$+252$




\subsection{Antecedentes en el español}

Según Majón Cabezas (2008), en las investigaciones de la madurez sintáctica en escolares de lengua española, podemos clasificar tres ámbitos territoriales hispanoamericanos: España, Puerto Rico y Chile. En España sobresalen los trabajos realizados por Torres González (1992) y posteriormente los de Torres López (2003) y Checa García (2004), entre otros; dichos trabajos se han centrado en el análisis de la madurez sintáctica a partir de textos escritos producidos por escolares. En Puerto Rico destacan los estudios de Rodríguez Fonseca (1991) y Espinet de Jesús (1996), que si bien realizan estudios de madurez sintáctica, están dirigidos a identificar variedad léxica. En Chile tenemos los aportes de Véliz (1987, 1988, 1999) y Muñoz y Véliz (1983), entre otros. A continuación, nos referiremos a aquellos que consideramos más pertinentes a la luz de nuestra investigación.

Los estudios de Mónica Véliz son pioneros en este tipo de investigaciones, por lo que han marcado la pauta de los trabajos posteriores (Torres González 1992). Véliz (1988) define la madurez sintáctica, siguiendo a Hunt (1970), como la habilidad para elaborar unidades sintácticas complejas desde el punto de vista estructural (Veliz 1988). El uso de los índices de madurez sintáctica de Hunt para la lengua inglesa y su adaptación al español se lo debemos a ella.

Véliz (1988) plantea dos hipótesis centrales: que las estructuras oracionales se hacen más complejas según se avanza en la edad cronológica

y mental; y que los índices primarios son medidas válidas y confiables de la madurez sintáctica para textos de escolares de habla española. Es así como, redefiniendo los índices de Hunt, entiende la unidad terminal como "una construcción constituida por una cláusula principal más las cláusulas subordinadas que puedan ir añadidas o incrustadas en ellas" (Veliz, 1988: 52), mientras que por cláusula entiende "una construcción libre o dependiente, articulada en sujeto y predicado, con el verbo en forma personal" (Véliz, 1988: 53).

Las conclusiones a las que llega en este trabajo son, en primer lugar, que se presentan diferencias en las estructuras sintácticas utilizadas por los escolares de los cursos estudiados, lo cual se expresa en la mayor complejidad y extensión de las unidades terminales y las cláusulas de los cursos superiores respecto de los inferiores. Además, la autora señala una notable disminución de la yuxtaposición y de la coordinación, mientras que la subordinación va creciendo significativamente a medida que aumenta el nivel escolar. Por otro lado, comprueba que el desarrollo 
El desarrollo de la sintaxis en la adolescencia: posibles influencias de naturaleza social / Carlos Balboa, Nina Crespo y Marcela Jonely de Lourdes Rivadeneira

sintáctico no termina al finalizar la enseñanza media (secundaria), sino que sigue avanzando. Asimismo, la autora identifica ciertos aspectos sintácticos que tardan en dominarse y cuyo aprendizaje se prolonga en el tiempo. En otras palabras, los escolares suelen utilizar ciertas estructuras, sin dominarlas completamente; por ejemplo, las cláusulas adjetivas introducidas por que son utilizadas desde cuarto básico (en la primaria), mientras que las introducidas por cual y cuyo pueden incluso ser recién reconocidas en los últimos años de enseñanza media.

Otra conclusión de suma importancia que saca la autora es el paralelismo entre la adquisición de la madurez sintáctica tanto en español como en inglés. En este sentido, las cláusulas relativas son los mejores indicadores de la madurez sintáctica en ambas lenguas, mientras que las cláusulas sustantivas dependen más de factores relacionados con el tipo de discurso que la madurez de los sujetos. Por último, una de las conclusiones más valiosas de Véliz (1988) es la comprobación de la validez y confiabilidad del índice de Hunt (1970), como instrumento de medición de la madurez sintáctica, para el estudio cuantitativo de la lengua escrita en sujetos distribuidos en un amplio rango de edad. Es así como señala que el mejor índice para medir la complejidad sintáctica es la longitud de la unidad terminal, luego sigue la longitud de la cláusula y, por último, el índice de cláusulas subordinadas por unidad terminal; a su vez, los índices secundarios más significativos son las cláusulas adjetivas por unidad -t, infinitivos por unidad -t, calificativos por unidad -t y frases preposicionales por unidad -t.

Una de las investigaciones más interesantes fue la realizada en conjunto por dos equipos de trabajo de la Universidad Chile y la Universidad de Concepción, presentado hace ya veinte años (Véliz et al. 1991). Los objetivos de esta investigación fueron evaluar el desarrollo sintáctico de textos escritos por estudiantes chilenos en el último año de enseñanza media e identificar de qué manera influyen las variables de nivel socioeconómico, régimen de estudios, regionalidad, sexo y modo de discurso. Los resultados de esta investigación son sumamente interesantes: básicamente, se señala que la hipótesis planteada sobre el nivel socioeconómico y el régimen de estudios no se cumple, puesto que, a diferencia de lo que se puede creer, los porcentajes más altos ocurren en aquellos escolares de menos recursos y en los de establecimientos educacionales públicos.

Otra de las conclusiones obtenidas fue que los escolares pasan por varias etapas; en una de ellas se utilizan los mecanismos de subordinación sin control, realizando oraciones muy extensas y complicadas.

$+254$


Además, "los índices sintácticos deben considerarse sólo como indicaciones técnicas de la estructuración sintáctica de los discursos" (Véliz et al. 1991: 80) y ese es el concepto que debe tenerse sobre madurez sintáctica, porque el aumento de los índices no siempre equivale a un discurso cualitativamente mejor. Por último, las conclusiones que se obtienen al considerar el modo de discurso son las más relevantes en esta investigación, pues las composiciones expositivas y argumentativas alcanzan una mayor complejidad sintáctica que las narrativas.

\section{Hipótesis}

Basándose en la propuesta de Bernstein $(1974,1989)$, autores como Bereiter et al. (1966), Bereiter y Engelmann (1966), Deutsch et al. (1967), Deutsch, Katz y Jensen (1968) y Whiteman y Deutsch (1968) proponen la idea del déficit, la que sostiene que el fracaso escolar de los sujetos de estratos más bajos obedece a una suerte de déficit lingüístico, en comparación con aquellos sujetos pertenecientes a los estratos medios y altos, por lo que se considera que el registro de los hablantes de estratos sociales bajos es erróneo o empobrecido. En relación a lo anterior, algunos autores (Labov 1969; Almeida 2003; López Morales 1993; Moreno Fernández 1998) han señalado que esto no puede diferenciarse en términos de mejor o peor manejo de la lengua, vale decir, los rasgos peculiares que tienen los estratos más bajos no deben considerarse inferiores o más pobres que la de los estratos medios y altos, sino variantes de una misma forma. De esta manera, las diferencias entre estas dos variantes diastráticas no deberían plantearse en términos de complejidad, sino de diversidad. A partir de allí, nuestra hipótesis apunta a que las producciones narrativas orales de adolescentes de estratos socioeconómicos bajos y altos no tendrán diferencias significativas en cuanto a mayor o menor complejidad sintáctica entre ellas. Es así como, según nuestra propuesta, la variable clase social no incide en la complejidad sintáctica de las narraciones orales producidas por adolescentes.

\section{Metodología}

Nuestra investigación se enmarca en los estudios correlacionales, puesto que se establece la relación entre la variable clase social y la variable complejidad sintáctica en narraciones orales producidas por adolescentes chilenos. Nuestro propósito fue estudiar cómo la variable clase social, es decir, la variable independiente en nuestra investigación, incide o no 
El desarrollo de la sintaxis en la adolescencia: posibles influencias de naturaleza social / Carlos Balboa, Nina Crespo y Marcela Jonely de Lourdes Rivadeneira

en la complejidad sintáctica de las narraciones orales producidas por jóvenes de estrato socioeconómico bajo y alto, la cual corresponde a la variable dependiente. Es en este sentido que nuestra investigación se enmarca dentro de un diseño no experimental.

\subsection{Muestra}

Moreno Fernández (1998) postula que -dado que las clases sociales son constructos sociológicos para caracterizar a los individuos de una sociedad y no cualidades intrínsecas a estos- la identificación de las distintas clases sociales para realizar estudios sociolingüísticos se realiza de acuerdo a ciertos índices. En nuestro caso, se consideró la clasificación del Ministerio de Educación de Chile (MINEDUC), basándose en criterios formulados en 2003, que agrupa los establecimientos en cuatro categorías. Como criterio se empleó el estrato socioeconómico (ingreso familiar) y grado de escolaridad de los padres de alumnos que rinden la prueba SIMCE (Sistema de Medición de la Calidad de la Educación). Cinco son los grupos, que se ordenan de menor a mayor, en cuanto a nivel socioeconómico: A, B, C, D, y E.

En función de esto, cabe señalar que la muestra consistió en alumnos de segundo año medio (de aproximadamente 15 años) de establecimientos educacionales municipales, que se encuentran en el grupo B (estrato socioeconómico bajo, ubicación urbana), y alumnos de establecimientos particulares, correspondientes al grupo E (estrato socioeconómico alto, ubicación urbana). Para controlar otras variables, como el nivel de instrucción, se consideró que los alumnos hubieran obtenido un promedio de entre 270 y 330 puntos en la prueba aplicada por el Sistema Nacional de Evaluación de resultados de aprendizaje del Ministerio de Educación de Chile (SIMCE). Dicho promedio los situaría entre los establecimientos que han logrado los puntajes dentro del tercio más alto de los obtenidos en la prueba. Asimismo, los colegios para la toma de la muestra son mixtos y poseen niveles de enseñanza básica y media. La muestra fue de un total de 60 individuos, 30 del grupo B y 30 del grupo E; en ambos grupos había 15 estudiantes hombres y 15 estudiantes mujeres, respectivamente.

\subsection{Procedimiento de recolección de los datos}

Se decidió utilizar como modalidad discursiva de análisis la narración, puesto que es una de las más tempranas en ser desarrollada, siendo la forma primaria de representación (Pavez, Coloma y Maggiolo 2008),

$+156$


por lo que en la adolescencia se espera que su dominio se encuentre completamente logrado. Para elicitar las narraciones orales producidas por los adolescentes, se utilizó como instrumento el corto sin diálogos "Fly Guy", creado por Simon Tofield (2010). Luego de que cada sujeto vio de modo individual el corto animado, realizó una narración oral del mismo, la cual fue grabada y luego transcrita.

\subsection{Procedimiento de análisis}

El análisis se inició con un conteo de palabras por texto producido, con la idea de dar cuenta de la fluidez que mostraban los sujetos. Posteriormente, se procedió -siguiendo a Veliz (1988) - a segmentar cada uno de los textos en las unidades -t constituyentes, es decir, en construcciones constituidas por una cláusula principal más las cláusulas subordinadas que pueden ir incluidas en ella (Véliz 1988). Asimismo, se identificaron las cláusulas, es decir, aquellas construcciones sintácticamente libres o dependientes, articuladas en sujeto y predicado y con el verbo en forma personal, clasificándolas en sustantivas, adjetivas y adverbiales. A continuación, se procedió a calcular los índices principales y algunos de los secundarios planteados por Véliz (1988). Los principales incluyeron: a) promedio de longitud en palabras de la unidad mínima terminal o unidad -t, considerado como el más útil indicador del crecimiento de la complejidad sintáctica por estar altamente correlacionado con la densidad sintáctica (López Morales 1991); b) promedio de longitud en palabras de cláusula; y c) índice de subordinación o número promedio de cláusulas por unidad mínima terminal, el cual indica la frecuencia de cláusulas presentes en cada unidad -t y da mejor cuenta de los factores que contribuyen intrínsecamente a hacer más compleja la sintaxis (Véliz 1988). Los secundarios que Véliz (1988) denomina clausales incluyen la proporción de cláusulas adjetivas por unidad -t, de sustantivas por unidad - $t$ y de adverbiales por unidad -t.

\section{Análisis de resultados}

A continuación daremos cuenta de tres resultados: por un lado el promedio de palabras por texto, los índices primarios de complejidad sintáctica

y los índices secundarios de complejidad sintáctica, de acuerdo con lo propuesto por Véliz (1988). 
El desarrollo de la sintaxis en la adolescencia: posibles influencias de naturaleza social / Carlos Balboa, Nina Crespo y Marcela Jonely de Lourdes Rivadeneira

\subsection{Palabras por texto}

Si bien el solo hecho de que un texto tenga mayor cantidad de palabras no mide mayor complejidad del mismo, en las producciones textuales de adolescentes, nos llama la atención que durante nuestra investigación identificamos que las narraciones elaboradas por sujetos de estratos socioeconómicos altos son, ostensiblemente, más extensas que las narraciones de estratos socioeconómicos bajos ( $48 \%$ de diferencia entre un grupo y otro). Esto nos lleva a concluir que los sujetos de estrato socioeconómico alto elaboran narraciones mucho más fluidas o de mayor variabilidad léxica, pero no implica que necesariamente sean más complejas.

\section{2. Índices de complejidad sintáctica}

En la Tabla 1 se pueden observar los resultados promedios y la desviación estándar de los grupos en cada uno de los índices primarios:

Tabla 1. Medidas descriptivas de promedio de longitud de unidad mínima terminal, de cláusula y de cláusulas por unidad -t según estrato socioeconómico y género

\begin{tabular}{|c|c|c|c|c|c|}
\hline \multirow[t]{2}{*}{ Índice } & \multirow[t]{2}{*}{ Género } & \multicolumn{2}{|c|}{ Estrato bajo } & \multicolumn{2}{|c|}{ Estrato alto } \\
\hline & & Promedio & Desv. Est. & Promedio & Desv. Est. \\
\hline \multirow{3}{*}{$\mathrm{PAL} / \mathrm{UT}$} & Femenino & 9,4 & 4,0 & 8,6 & 1,7 \\
\hline & Masculino & 7,9 & 1,5 & 8,7 & 1,7 \\
\hline & Total & 8,7 & 3,1 & 8,6 & 1,7 \\
\hline \multirow{3}{*}{$\mathrm{PAL} / \mathrm{CL}$} & Femenino & 10,04 & 4,8 & 6,38 & 0,71 \\
\hline & Masculino & 5,67 & 0,72 & 6,11 & 0,86 \\
\hline & Total & 7,85 & 4,11 & 6,24 & 0,80 \\
\hline \multirow{3}{*}{ CL/UT } & Femenino & 1,65 & 0,8 & 1,34 & 0,2 \\
\hline & Masculino & 1,41 & 0,3 & 1,41 & 0,3 \\
\hline & Total & 1,53 & 0,6 & 1,37 & 0,2 \\
\hline
\end{tabular}

Como podemos observar, tanto el promedio de longitud de la unidad mínima terminal, como el de la longitud de las cláusulas y de las cláusulas por unidad -t es mayor en las narraciones elaboradas por estudiantes de estrato socioeconómico bajo. Es decir, estos últimos son capaces de elaborar con mayor complejidad sintáctica sus narraciones. Esto se refiere especialmente a las mujeres del estrato bajo, cuyos puntajes destacan en forma evidente respecto de sus pares de estrato alto.

Por otra parte, los resultados obtenidos por estudiantes de estrato socioeconómico alto, desde una perspectiva intragrupal, son más ho-

$+258$


mogéneos en los tres índices, mientras que en el grupo de mujeres de estrato bajo se observa un número de estudiantes cuyos puntajes escapan de la media de su grupo introduciendo gran variabilidad. Esto podemos observarlo en el Gráfico 1 y el Gráfico 2:

Gráfico 1. Distribución de puntajes intragrupales por longitud de unidad -t y cláusula

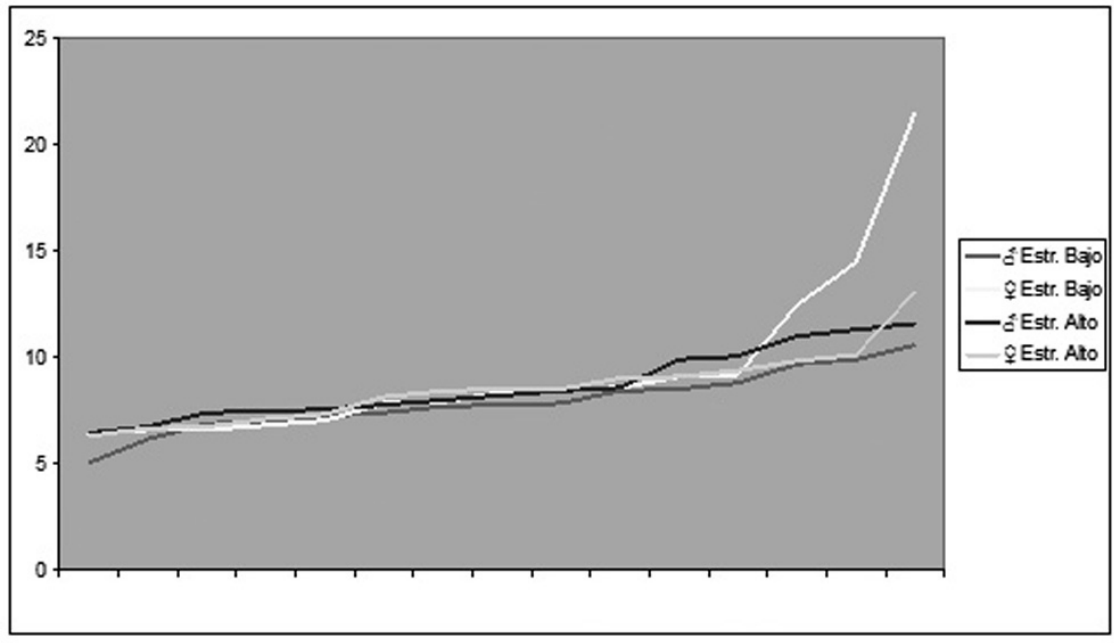

Gráfico 2. Distribución de puntajes intragrupales por cláusulas/unidad -t

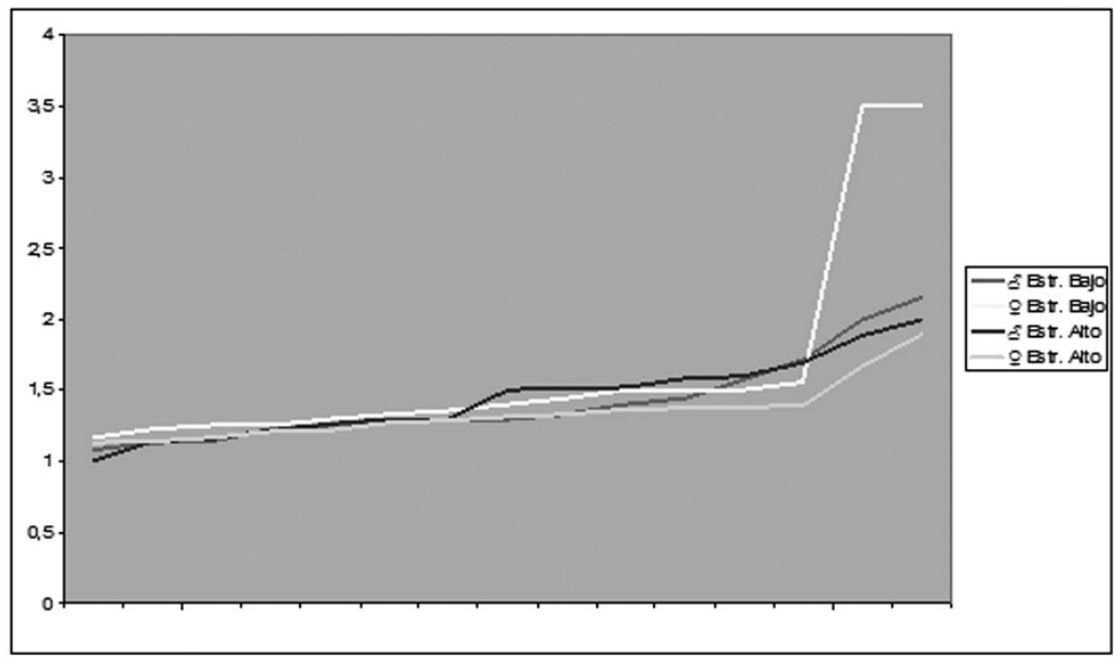

Para observar si la diferencia que favorece al estrato bajo es significativa, fue necesario aplicar pruebas estadísticas a los datos. Los resultados de la prueba de Kruskal-Wallis aplicada a dichos datos nos indica que no existen diferencias significativas en el promedio de longitud de la unidad mínima terminal $\left(\chi^{2}=0.6733\right)$, tampoco en el promedio de longitud de 
El desarrollo de la sintaxis en la adolescencia: posibles influencias de naturaleza social / Carlos Balboa, Nina Crespo y Marcela Jonely de Lourdes Rivadeneira

cláusula $\left(\chi^{2}=0.6733\right)$, ni en el de índice de subordinación $\left(\chi^{2}=1.012\right.$ valor), ya que todos estos valores son superiores a $p<0,05$. De estos resultados, puede inferirse que las narraciones orales de estudiantes de segundo año medio, provenientes de estratos socioeconómicos bajo y alto no tienen diferencias en términos de complejidad sintáctica.

\section{3. Índices secundarios de complejidad sintáctica}

En la Tabla 2 se observan los resultados promedio y la desviación estándar de los grupos en cada uno de los índices secundarios:

Tabla 2. Medidas descriptivas de promedio de índice de cláusulas adjetivas, cláusulas sustantivas y cláusulas adverbiales por unidad -t según estrato socioeconómico y género

\begin{tabular}{|c|c|c|c|c|c|}
\hline \multirow{2}{*}{ Índice } & \multirow[t]{2}{*}{ Género } & \multicolumn{2}{|c|}{ Estrato bajo } & \multicolumn{2}{|c|}{ Estrato alto } \\
\hline & & Promedio & Desv. Est. & Promedio & Desv. Est. \\
\hline \multirow{3}{*}{ CLAdj/UT } & Femenino & 0,3 & 0,6 & 0,1 & 0,1 \\
\hline & Masculino & 0,1 & 0,1 & 0,2 & 0,1 \\
\hline & Total & 0,2 & 0,4 & 0,1 & 0,1 \\
\hline \multirow{3}{*}{ CLASust/UT } & Femenino & 0,2 & 0,2 & 0,2 & 0,1 \\
\hline & Masculino & 0,2 & 0,2 & 0,2 & 0,2 \\
\hline & Total & 0,2 & 0,2 & 0,2 & 0,2 \\
\hline \multirow{3}{*}{ CLAdv/UT } & Femenino & 0,1 & 0,2 & 0,1 & 0,1 \\
\hline & Masculino & 0,1 & 0,1 & 0,08 & 0,1 \\
\hline & Total & 0,1 & 0,1 & 0,09 & 0,1 \\
\hline
\end{tabular}

En este caso, podemos observar que no se presentan diferencias muy evidentes, tanto a nivel grupal como intragrupal en relación a dos de los tres índices analizados. Es así como mientras la proporción de cláusulas sustantivas y adverbiales por unidad -t apenas exhibe diferencias mínimas de puntaje, en el caso de las cláusulas adjetivas es el estrato bajo el que presenta los puntajes más altos y, en especial, las mujeres. El test de prueba de hipótesis Kruskal-Wallis permitió corroborar que no existen diferencias significativas en el índice promedio de cláusulas adjetivas por unidades - $t\left(\chi^{2}=0.1778\right)$, tampoco en el de sustantivas por unidad -t $\left(\chi^{2}=0.1778\right)$, ni en las adverbiales $\left(\chi^{2}=1.6837\right)$, ya que ninguno de los valores fue menor a $\mathrm{p}<0,05$.

\section{Conclusiones y discusión}

Ya se señaló que la teoría del déficit, basada en Bernstein (1974, 1989), sostenía que los sujetos de estrato bajo, al utilizar el código restringido,

$+260$


manifiestan un habla cuya organización sintáctica será limitada y que, por lo tanto, sería inferior a la de los estratos altos (Bereiter et al. 1966; Bereiter y Engelmann 1966; Deutsch et al. 1967; Deutsch, Katz y Jensen 1968; Whiteman y Deutsch 1968). Los resultados de este trabajo no parecen avalar dicha teoría, ya que, si bien los sujetos de estrato alto producen textos evidentemente más extensos que los de estrato bajo, no hay diferencias significativas en cuanto a la complejidad sintáctica. En este sentido, puede decirse que los primeros hablan más y parecen más cómodos con el uso de la palabra que sus pares de estratos menos favorecidos. Por otra parte, y aunque escapa a los objetivos de este estudio, cabe consignar diferencias a nivel de ciertos rasgos fonéticos, como la tendencia de los hablantes de estrato socioeconómico bajo a cerrar vocales como en dispertar o el uso de /ch/ fricativa. Sin embargo, ninguna de estas variantes permitiría hablar objetivamente de mayor o menor riqueza lingüística.

Los hallazgos de nuestra investigación coinciden con el trabajo pionero de Labov (1969), quien se opuso al concepto de "privación verbal" que explicaba la variación lingüística entre sujetos de distintos estratos sociales que vivían en la ciudad de Nueva York. El investigador encuentra también que los sujetos de estratos altos y medios utilizan más vocabulario y producen en general textos más extensos y ampulosos, pero que, desde el punto de vista objetivo, no son más complejos que los producidos por sus pares de estratos menos favorecidos. Asimismo, Véliz et al. (1991) observan mayores valores en los textos escritos elaborados por sujetos de estrato socioeconómico bajo, por sobre los valores obtenidos de los textos elaborados por sujetos del estrato socioeconómico alto. De igual forma, los autores señalan que las diferencias encontradas entre los niveles socioeconómicos bajo, medio y alto no son significativas estadísticamente (Véliz et al. 1991).

De esta forma, es posible afirmar que lo que diferencia a las formas de habla de estudiantes pertenecientes a estratos socioeconómicos bajos y altos en Chile, en lo que se refiere a la sintaxis, es la variabilidad sociolingüística. En este sentido, la manera de hablar que poseen los sujetos de estrato bajo, por más que puedan ser menos valoradas e incluso considerarse estereotipos, no es objetivamente inferior a aquella producida por sus pares de estratos altos. Así, las variantes lingüísticas que se relacionan con la clase social deben ser interpretadas en el marco de una visión sociológica, más relacionada con la identidad grupal que con la corrección lingüística. De igual forma, muchas de las variaciones diastráticas del lenguaje deben ser consideradas como significados socia- 
El desarrollo de la sintaxis en la adolescencia: posibles influencias de naturaleza social / Carlos Balboa, Nina Crespo y Marcela Jonely de Lourdes Rivadeneira

les (Almeida 2003) que les permiten a las personas marcar su pertenencia a un entorno social que los valida como individuos.

Otro punto interesante relacionado con el variacionismo sociolingüístico está vinculado con el comportamiento lingüístico de las mujeres, puesto que se observó que, en las narraciones elaboradas por el grupo de mujeres de estrato socioeconómico bajo, ciertas muestras se escapaban del puntaje promedio. Este fenómeno, que podríamos considerar como totalmente vano o anecdótico, según algunas investigaciones (SilvaCorvalán 1989; Fasold 1990; López Morales 1992, 1993; Blas Arroyo 1999; Tusón 1999, Trudgill 2000), se explicaría a partir de la noción de que las mujeres poseen actitudes lingüísticas más conservadoras y más apegadas a la noción de prestigio. Esto correspondería a lo que Labov (2001) denomina "la paradoja del género", por cuanto las mujeres se ajustan más que los hombres a las normas establecidas de la sociedad, pero las obedecen menos que los hombres cuando tales normas no existen abiertamente.

Finalmente, cabe señalar dos limitaciones de este trabajo. Una de ellas está relacionada con el nivel de instrucción de los hablantes, ya que, si bien los sujetos pertenecían a distintos estratos, evidenciaban en las medidas oficiales un nivel de instrucción similar. Por ende, sería necesario observar cómo afecta la instrucción a la complejidad lingüística cuando el rasgo social está acompañado de diferencias a nivel de formación educativa, lo cual permitiría entrever el rol que juega la escolarización en el desarrollo tardío del lenguaje. La otra limitación está relacionada con el contexto estilístico de los discursos elicitados. Labov (1972) y Rissel (1981), entre muchos otros, ya señalaban que, cuando se trata con variación sociolingüística, hay que considerar el tema del estilo. En este sentido, los textos elaborados por nuestros alumnos corresponden a un estilo académico elaborado y formal, porque la tarea de elicitación fue planteada de manera similar a una tarea académica. Debido a esto, es posible considerar que el proceso de literacidad propio del ámbito escolar ha igualado las formas lingüísticas elegidas por los hablantes. Una comparación legítima de la complejidad sintáctica en los textos producidos por hablantes de distinto estrato socioeconómico debe considerar también producciones de habla espontánea e informal.

Por otra parte, el discurso narrativo se caracteriza por la predominancia de ciertas formas subordinadas -como la sustantiva-, por lo que sería interesante observar qué ocurre cuando alumnos de poblaciones similares elaboran textos argumentativos o expositivos, tanto en el tipo

$+262$


de subordinada que pudieran preferir, como en las posibles variaciones lingüísticas que pudieran surgir, debido a las diferencias sociales de los hablantes.

A partir de allí, puede considerarse este trabajo como exploratorio y preliminar, y sus hallazgos deberían ser corroborados con estudios posteriores, en los cuales se utilizara una muestra de sujetos estadísticamente más representativa que permitiera, por un lado, conocer a fondo la naturaleza de las diferencias diastráticas intergrupales y, por otro, dar cuenta de las diferencias intergrupales que consideran la variable género. Creemos que esta línea de investigación sería fructífera para comprender la dinámica entre el sistema de posibilidades que implica la lengua y las elecciones que el individuo realiza para construir y construirse a sí mismo en el marco de una sociedad compleja.

\section{Bibliografía}

Almeida, M. (2003). Sociolingüística. Tenerife: Universidad de La Laguna.

Barrera, L. \& L. Fraca de Barrera (2004). Psicolingüística y desarrollo del español. Caracas: Monte Ávila.

Bereiter, C. et al. (1966). "An academically oriented pre-school for culturally deprived children", in F. M. Hechinger (ed.), Pre-school Education Today. New York: Doubleday, 105-137.

\& S. Engelmann (1966). Teaching Disadvantaged Children in the Pre-school. New Jersey: Prentice May.

Berman, R. (2000). "Children's innovative verbs vs nouns: Structured elicitations and spontaneous coinages", in L. Menn $\&$ N. Bernstein-Ratner (eds.), Methods for studying language production. Mahwah, NJ: Erlbaum, 69-93.

Bernstein, B. (1971). Class, codes and control: theoretical studies towards a sociology of language. Londres: Routledge \& Kegan Paul.

(1974). "Códigos amplios y restringidos: sus orígenes sociales y algunas consecuencias", en Garvin, P. \& Lastra de Suárez, Y. (eds.) Antología de estudios de etnolingüísticay sociolingüistica. Ciudad de México: UNAM, 357-374.

(1985). "Clase social, lenguaje y socialización". Educación y Sociedad 4: 129-146. 
El desarrollo de la sintaxis en la adolescencia: posibles influencias de naturaleza social / Carlos

Balboa, Nina Crespo y Marcela Jonely de Lourdes Rivadeneira

(1989). Clases, códigos y control 1. Estudios teóricos para una sociología del lenguaje. Madrid: Akal.

Blas Arroyo, J. L. (1999). "Las actitudes hacia la variación intradialectal en la sociolingüística hispánica". Estudios Filológicos 34: 47-72.

Cedergren, H. \& D. Sankoff (1974). "Variable rules: Performance as a statistic reflection of competence". Language 50: 333-355.

Checa, I. (2004). "Medidas de madurez sintáctica aplicadas a lecturas de ELE”. Interlingüística 16: 1-15.

\& C. Lozano (2002). "Los índices de madurez sintáctica de Hunt a la luz de las distintas corrientes generativistas". Interlingüística13: 336-358.

Coseriu, E. (1973). Teoría del lenguaje y lingüistica general. Madrid: Gredos.

Deutsch, M. et al. (1967). The disadvantaged child: studies of the social environment and the learning process. New York/ London: Basic Books.

; I. Katz \& A. R. Jensen (1968). Social class, race and psychological development. New York: Holt.

Duek, C. \& G. Inda (2006). "La teoría de la estratificación social de Weber: un análisis crítico". Revista Austral de Ciencias Sociales 11: 05-24.

Espinet de Jesús, L. (1996). "Índices primarios de madurez sintáctica en escritores profesionales puertorriqueños: variables sexo y modo del discurso". REALE 6: 37-52.

Fasold, R. (1990). The sociolinguistics of languaje. Oxford: Blackwell.

Fernández, P. (1994). "La evolución de la teoría de los códigos sociolingüísticos en B. Bernstein”. Didáctica 6: 99112.

Gil, J. M. (2001). Introducción a las teorías lingüísticas del siglo xx. Buenos Aires: Melusina.

Junco, M. (1999). "La evaluación del discurso escrito narrativo en el nivel elemental de enseñanza". Milenio 3: 213-244.

Katzenberger, I. (2004). "The development of clause packaging in spoken and written texts". Journal of Pragmatics 36.10: 19211948.

164


Hunt, K. (1970). "Syntactic maturity in school children and adults". Monographs of the Society for Research in Child Development 35.3: 3-67.

Labov, W. (1965). "On the mechanism of linguistic change". En Georgetown Monographs on Language and Linguistics, 18:91-114.

(1966). The social stratification of English in New York City. Washington, DC: Center for Applied Linguistics.

(1969). "A note on the relation of reading failure to peer group status in urban ghettos". The Teacher's College Record 70.5: 395-405.

(1972). Sociolinguistic patterns. Pennsylvania: University of Pennsylvania Press.

(1994). Principles of linguistic change. Vol. 1: Internal factors. Oxford: Basil Blackwell.

(2001). Principles of linguistic change. Vol. 2: Social factors. Oxford: Basil Blackwell.

\& G. Sankoff (1985). "Variation theory". Paper presented at NWAV XIV, Georgetown University, Washington, DC.

Lavandera, B. (1984). Variación y significado. Buenos Aires: Hachette.

Lewandowski, T. (1995). Diccionario de lingüística. Madrid: Cátedra.

López, H. (1984). Enseñanza de la lengua materna (lingüística para maestros de español). Madrid: Playor.

(1992). El español del Caribe. Madrid: Editorial Mapfre.

(1993). Sociolingüística. Madrid: Gredos.

Makuc, M. (2008). "Un caso de variación sintáctico-semántica del verbo en el español de Magallanes: una aproximación sociolingüística”. Magallania 36.2: 79-90.

Manjón-Cabeza, A. (2008). "Algunas observaciones a las técnicas de medición de riqueza sintáctica". Comunicación presentada en XV Jornadas sobre la Lengua Española y su Enseñanza el Español en Contexto, Universidad de Granada: Facultad de Filosofía y Letras.

Miller, G. A. (1962). "Some psychological studies of grammar". American Psychologist 17.748-62.

Ministerio de Educación de Chile. (2003). Metodología para agrupar establecimientos por nivel socioeconómico. División de 
El desarrollo de la sintaxis en la adolescencia: posibles influencias de naturaleza social / Carlos Balboa, Nina Crespo y Marcela Jonely de Lourdes Rivadeneira

Planificación y Presupuesto, Departamento de Estudios y Estadisticas. Disponible online http://www.mineduc. cl/biblio/documento/396_Construccion_de_grupos_ comparables_de_establecimientos_SIMCE_2.pdf.

Moreno, F. (1998). Principios de sociolingüística y sociología del lenguaje. Barcelona: Ariel.

Muñoz, G. \& M. Véliz (1983). "Incidencia de combinación de oraciones en la madurez sintáctica y en la calidad general de la composición”. RLA 21: 81-86.

Nir, B. \& R. A. Berman (2010). "Complex syntax as a window on contrastive rhetoric". Journal of Pragmatics 42: 744-765.

Oyanedel, M. \& J. L. Samaniego (1998-1999). "Notas para un nuevo perfil lingüístico del español de Santiago de Chile", Boletín de Filología de la Universidad de Chile 37.2: 899-913.

Pávez, M. M.; L. Martínez \& C. Coloma (1997). "The structure of conversation in mentally retarded children". Clinical Linguistics \& Phonetics 11.6: 455-466.

; C. Coloma \& M. Maggiolo (2008). El desarrollo narrativo en niños. Una propuesta práctica para la evaluación y la intervención en niños con trastorno del lenguaje. Barcelona: Ars Médica.

Rissel, D. (1981). "Diferencias entre el habla femenina y la masculina en español". Thesaurus 36: 305-322.

Rodríguez, L. (1991). "Índices de madurez sintáctica en escolares puertorriqueños de escuela primaria", en López, $\mathrm{H}$. (ed.) La enseñanza del español como lengua materna. Actas del II Seminario Internacional sobre Aportes de la lingüística a la enseñanza del español como lengua materna, Río Piedras, Puerto Rico, 133-143.

Rona, J. P. (1974). "La concepción estructural de la sociolingüística”, en Garvin, P. \& Lastra de Suárez, Y. (eds.) Antología de estudios de etnolingüística y sociolingüística, Ciudad de México: UNAM, 216-230.

Sáez, L. (2000). [1999]. "El español de Chile en las postrimerías del siglo XX", en Sáez, L., Cómo hablamos los chilenos. Ocho aproximaciones. Santiago de Chile. [Ediciones del Bachillerato en Ciencias y Humanidades, Universidad de Santiago de Chile].

166


Sankoff, D. (1978). Linguistic variation: models and methods. New York: Academia Press.

(1988a). "Problems of representativiness", in Cohen, J. et al. (eds.) Sociolinguistics: an internacional handbook of the science of language and society. Amsterdam: North Holland/ Warsaw: Polish Scientific, 677-689.

Silva-Corvalán, C. (1980-1981). "La función pragmática de la duplicación de pronombres clíticos". Boletín de Filología de la Universidad de Chile. Homenaje a Ambrosio Rabanales 31: 561-570.

(1989). Sociolingüística: Teoría y análisis. Madrid: Alhambra.

(2001). Sociolingüística y pragmática del español. Washington: Georgetown University.

Tofield, S. (2010). "The Fly Guy". Video corto, disponible online http:// www.simonscat.com/flyguy.html.

Torres, A. (1992). Madurez sintáctica en estudiantes no universitarios de la zona metropolitana de Tenerife. Tesis Doctoral: Universidad de la Laguna.

Torres, M. C. (2003). "Estudio comparativo de complejidad sintáctica en alumnos de Granada", en Moya Corral y Montoya Ramírez (eds.). Variación lingüística y enseñanza de la lengua española. Actas de las VIII Jornadas sobre la Enseñanza de la Lengua Española, Universidad de Granada, 447-472.

Tusón, A. (1999). "Diferencia sexual y diversidad lingüística", en Lomas, C. (coord.). iIguales o diferentes?: Género, diferencia sexual, lenguaje y educación, Barcelona: Paidós, 85-100.

Trudgill, P. (2000). Sociolinguistics: an introduction to language and society. London: Penguin Books. [4th edition].

Trujillo, R. (1996). Principios de semántica textual. Madrid: ArcoLibros.

Véliz, M. (1987). "Protosubordinadores: formas de transición en el proceso de desarrollo sintáctico". RLA 25: 45-53.

(1988). "Evaluación de la madurez sintáctica en el discurso escrito". RLA 26: 105-141.

(1999). "Complejidad sintáctica y modo del discurso". Estudios Filológicos 34: 181-192. 
El desarrollo de la sintaxis en la adolescencia: posibles influencias de naturaleza social / Carlos Balboa, Nina Crespo y Marcela Jonely de Lourdes Rivadeneira

; G. Muñoz \& M. Echeverría. (1985). "Madurez sintáctica y combinación de oraciones en estudiantes universitarios". RLA 23: 107-119.

et al. (1991). "Evaluación de la madurez sintáctica en estudiantes chilenos de cuarto medio". Estudios Filológicos 26: 71-81.

Weinreich, U.; W. Labov and M. Herzog (1968). "Empirical foundations for a theory of language change." In Winfred Lehmann and Yakov Malkiel (eds.), Directions for historical linguistics: A symposium. Austin: University of Texas Press. 95-188.

Whiteman, M. \& M. Deutsch (1968). "Social disadvantage as related to intellective and language development", in Deutsh, Katz y Jensen (eds.) Social class, race and psychological development. New York: Holt.

$+168$

